\title{
CONSTRUCTION OF SOLITON AND MULTIPLE SOLITON SOLUTIONS TO THE LONGITUDINAL WAVE MOTION EQUATION IN A MAGNETO-ELECTRO-ELASTIC CIRCULAR ROD AND THE DRINFELD-SOKOLOV-WILSON EQUATION
}

\author{
MD NUR ALAM AND CEMIL TUNC
}

Received 25 November, 2019

\begin{abstract}
The present paper implements the novel generalized $\left(G^{\prime} / G\right)$-expansion approach in solving the most popular nonlinear wave equations such as the longitudinal wave motion equation in a magneto-electro-elastic circular rod and the Drinfeld-Sokolov-Wilson equation. In this regard, we investigate the method to obtain new type of wave solutions of the studied models. New exact wave solutions are derived in the structures such as singular bright solition, compaction, singular bright periodic wave solitions, singular dark solition and singular dark periodic wave solition solutions of the studied models by using the novel generalized $\left(G^{\prime} / G\right)$-expansion scheme. To draw the physical aspect of the got results, the 2D, 3D surfaces as well as the relating the contour plot surfaces of some acquired results are performed. The obtained results can assist to illustrate the physical application of the examined models and other nonlinear physical models appearing in mathematical physics.
\end{abstract}

2010 Mathematics Subject Classification: 35C07; 35C08; 35Q53

Keywords: novel generalized $\left(\frac{G \prime}{G}\right)$-expansion method, the longitudinal wave motion equation in a magneto-electro-elastic circular rod, the Drinfeld-Sokolov-Wilson equation, exact solutions

\section{INTRODUCTION}

It can be said with certainty, there are various nonlinear physical phenomena in nature that are represented by nonlinear evolution equations (NLEEs). Under deliberation prompt construction of symbolic computation operations, the exploration for the exact solutions of nonlinear evolutions has explored a lot of attention because exact solutions can explain the physical property of a natural system properly. Each exact solution of a system of NLEEs corresponds to a particular process. Therefore, methods for obtaining explicit exact solutions of nonlinear equations of partial differential equations (PDEs) implement a significant function in applied mathematics,

The authors would like to acknowledge CAS-TWAS presidents fellowship program. 
mathematical physics and engineering. In the last two decades, a variety of procedures have been introduced and deployed to the nonlinear equations of partial differential equations, including the $\exp (-\phi(\xi))$-expansion method $[9,10,12]$, the sineGordon expansion method [17, 19], the Cole-Hopf transformation method [49], the improved Bernoulli sub-equation function method [15], the modified $\exp (-\phi(\xi))$ expansion method [16,41], F-expansion method [43,59], the tanh-sech method [35, 48], sine-cosine method [47,55], the $\left(G^{\prime} / G\right)$-expansion method [7,32,46,57,58], the extended trial equation method $[38,44]$ and so on.

Naher and Abdullah [39] introduced a novel generalized $\left(G^{\prime} / G\right)$-expansion method for the treatment of NLEEs. Afterwards, a few scientists and researchers examined some nonlinear equation of partial differential equations to construct exact solutions through this method. One might see references $[3,6,11]$ for better understanding. This paper will employ the novel generalized $\left(\frac{G \prime}{G}\right)$-expansion system that will show explicitly exact wave solutions as well as other solutions. The equation for the model interpreted here is a nonlinear equation. We observe that this model has not yet been examined employing the novel generalized $\left(\frac{G \prime}{G}\right)$-expansion system. Hence, this paper is designed as follows: In Sections 2 and 3, application of the novel generalized $\left(\frac{G \prime}{G}\right)$-expansion method to the longitudinal wave motion equation in a magneto-electro-elastic circular rod and the Drinfeld-Sokolov-Wilson equation is discussed and then we obtain soliton solutions. Subsequently, the conclusion is presented in the last section.

\section{EXACT SOLUTION OF THE LONGITUDINAL WAVE MOTION EQUATION IN A MAGNETO-ELECTRO-ELASTIC CIRCULAR ROD}

With the growing acceptance of magneto-electro-elastic structures in numerous engineering fields which as sensors, actuators, and so many interested various scientists and researchers. Waved propagation in MEE media also has attracted many researchers $([22,23,34,52])$. Very recently, Xue et al. [53] has discovered the nonlinear longitudinal wave equation including dispersion produced through the transverse Poisson's effect in an magneto-electro-elastic circular rod, and Jacobi elliptic function approach has easily received the travelling and solitary waves. The nonlinear longitudinal wave equation has the following form:

$$
\frac{\delta^{2} u}{\delta t^{2}}-c_{0}^{2} \frac{\delta^{2} u}{\delta z^{2}}-\frac{\delta^{2}}{\delta z^{2}}\left(\frac{c_{0}^{2}}{2} u^{2}+N \frac{\delta^{2} u}{\delta t^{2}}\right)=0 .
$$

If we choose $u=V, c_{0}=c, N=p$ and $Z=x$ for the sake of simplicity, we can rewrite this model as follows:

$$
\frac{\delta^{2} V}{\delta t^{2}}-c^{2} \frac{\delta^{2} V}{\delta x^{2}}-\frac{\delta^{2}}{\delta x^{2}}\left(\frac{c^{2}}{2} V^{2}+p \frac{\delta^{2} V}{\delta t^{2}}\right)=0
$$


here $p$ is the dispersion parameter and $c$ is the wave velocity for an magneto-electroelastic circular rod. The dispersion parameter and the wave velocity for an magnetoelectro-elastic circular rod depend on the material characteristics and also the geometry of the rod [53]. For the actual significance of equation (2.1) concluded that the infinite homogeneous magneto-electro-elastic circular rod is make of composite $\mathrm{BaTiO}_{3}-\mathrm{CoFe}_{2} \mathrm{O}_{4}$ with various volume fractions $\left(v_{f}\right) \mathrm{Of}_{\mathrm{BaTiO}}$. It might be also noted that the rod has a radius of $R=0.05 \mathrm{~m}$. The material features of the composite are obtained to make the simple rule of the mixture in accordance with the volume fraction. For more further details, the readers can refer to $[33,53,56]$. Numerous computational procedures have been employed to study the exact wave solutions of the linear longitudinal wave velocity for a MEE circular rod [16,20,26,33,36,42,44, $53,56]$. In this section, we firstly convert partial differential equation to the ordinary differential equation of the longitudinal wave motion equation in a magneto-electroelastic circular rod through the travelling wave equation. Then, we apply the novel generalized $\left(\frac{G \prime}{G}\right)$-expansion method to determine exact wave solution of the above model.

We assume that the equation (2.1) is in the following form:

$$
V(\xi)=V(x, t), \xi=k(x-\omega t),
$$

where $k$ and $\omega$ are the wave number and wave velocity, respectively.

Equation (2.2) and equation (2.1) convert into a nonlinear equation as follows:

$$
k^{2} \frac{d^{4} V}{d \xi^{4}}-\frac{\omega^{2}-c^{2}}{p \omega^{2}} \frac{d^{2} V}{d \xi^{2}}+\frac{c^{2}}{2 p \omega^{2}} \frac{d^{2}\left(V^{2}\right)}{d \xi^{2}}=0
$$

Integrating equation (2.3) twice with respect to $\xi$, and letting the integral constants be zero for convenience, we then have

$$
k^{2} \frac{d^{2} V}{d \xi^{2}}-\frac{\omega^{2}-c^{2}}{p \omega^{2}} V+\frac{c^{2}}{2 p \omega^{2}} V^{2}=0 .
$$

According to the newly generalized $\left(\frac{G \prime}{G}\right)$-expansion method $[3,6,11]$, we apply the balance rule in the equation (2.4), yields $n=2$. Therefore, we have

$$
V(\xi)=a_{0}+a_{1}\left(d+T(\xi)+a_{2}\left(d+T(\xi)^{2}+b_{1}(d+T(\xi))^{-1}+b_{2}(d+T(\xi))^{-2},\right.\right.
$$

where $T(\xi)=\left(\frac{G \prime}{G}\right)$ and the coefficients $a_{0}, a_{1}, a_{2}, b_{1}, b_{2}$ and $d$ are free unknown arbitrary constants to be evaluated. Plugging the values of equation (2.5) into equation (2.4) and then equating each coefficients of $(d+T(\xi))^{n}$ to zero, we get the following relations, respectively:

- Cluster one: $d=-\frac{B}{2 \Psi}, k^{2}=\frac{\omega^{2}-c^{2}}{\omega^{2}} \frac{A^{2}}{4 E p \Psi+B^{2} p}, \omega=\omega, A_{0}=-\frac{3\left(c^{2}-\omega^{2}\right)}{c^{2}}, A_{1}=0$, $A_{2}=0, B_{1}=0, B_{2}=\frac{3}{4} \frac{c^{2}-\omega^{2}}{c^{2}} \frac{4 E \Psi+B^{2}}{\Psi^{2}}$,

- Cluster two: $d=-\frac{B}{2 \Psi}, k^{2}=\frac{c^{2}-\omega^{2}}{\omega^{2}} \frac{A^{2}}{4 E p \Psi+B^{2} p}, \omega=\omega, A_{0}=\frac{\left(c^{2}-\omega^{2}\right)}{c^{2}}, A_{1}=0$, $A_{2}=0, B_{1}=0, B_{2}=-\frac{3}{4} \frac{c^{2}-\omega^{2}}{c^{2}} \frac{4 E \Psi+B^{2}}{\Psi^{2}}$, 
- Cluster three: $d=-\frac{B}{2 \Psi}, k^{2}=\frac{\omega^{2}-c^{2}}{\omega^{2}} \frac{A^{2}}{16 E p \Psi+4 B^{2} p}, \omega=\omega, A_{0}=-\frac{1}{2} \frac{\left(c^{2}-\omega^{2}\right)}{c^{2}}$, $A_{1}=0, A_{2}=-\frac{\left(c^{2}-\omega^{2}\right)}{c^{2}} \frac{\Psi^{2}}{4 E \Psi+B^{2}}, B_{1}=0, B_{2}=-\frac{3}{16} \frac{c^{2}-\omega^{2}}{c^{2}} \frac{4 E \Psi+B^{2}}{\Psi^{2}}$,

- Cluster four: $d=-\frac{B}{2 \Psi}, \quad k^{2}=\frac{\omega^{2}-c^{2}}{\omega^{2}} \frac{A^{2}}{16 E p \Psi+4 B^{2} p}, \quad \omega=\omega, \quad A_{0}=$ $-\frac{3}{2} \frac{\left(c^{2}-\omega^{2}\right)}{c^{2}}, A_{1}=0, A_{2}=\frac{3\left(c^{2}-\omega^{2}\right)}{c^{2}} \frac{\Psi^{2}}{4 E \Psi+B^{2}}, B_{1}=0, B_{2}=\frac{3}{16} \frac{c^{2}-\omega^{2}}{c^{2}} \frac{4 E \Psi+B^{2}}{\Psi^{2}}$,

- Cluster five: $d=\frac{1}{6 \Psi}\left(-3 B \pm \sqrt{3}\left(E \Psi-B^{2}\right)\right), \quad k^{2}=\frac{\omega^{2}-c^{2}}{\omega^{2}} \frac{A^{2}}{4 E p \Psi+B^{2} p}$, $\omega=\omega, \quad A_{0}=-\frac{2\left(c^{2}-\omega^{2}\right)}{c^{2}}, \quad A_{1}=0, \quad A_{2}=0, \quad B_{1}=\frac{2\left(4 E \Psi+B^{2}\right)\left(c^{2}-\omega^{2}\right)}{\Psi c^{2}\left( \pm \sqrt{3}\left(E \Psi-B^{2}\right)\right.}$, $B_{2}=\frac{1}{3} \frac{\left(4 E \Psi+B^{2}\right)\left(c^{2}-\omega^{2}\right)}{\Psi^{2} c^{2}}$,

- Cluster six: $\quad d=\frac{1}{6 \Psi}\left(-3 B \pm \sqrt{3}\left(E \Psi-B^{2}\right)\right), \quad k^{2}=\frac{\omega^{2}-c^{2}}{\omega^{2}} \frac{A^{2}}{4 E p \Psi+B^{2} p}$, $\omega=\omega, \quad A_{0}=0, \quad A_{1}=0, \quad A_{2}=0, \quad B_{1}=-\frac{2\left(4 E \Psi+B^{2}\right)\left(c^{2}-\omega^{2}\right)}{\Psi c^{2}\left( \pm \sqrt{3}\left(E \Psi-B^{2}\right)\right.}$, $B_{2}=-\frac{1}{3} \frac{\left(4 E \Psi+B^{2}\right)\left(c^{2}-\omega^{2}\right)}{\Psi^{2} c^{2}}$,

- Cluster seven: $d=d, k^{2}=\frac{\omega^{2}-c^{2}}{\omega^{2}} \frac{A^{2}}{4 E p \Psi+B^{2} p}, \omega=\omega, A_{0}=\frac{c^{2}-\omega^{2}}{c^{2}} \frac{d^{2} \Psi^{2}+B d \Psi-E \Psi}{4 E \Psi+B^{2}}$, $A_{1}=0, \quad A_{2}=0, \quad B_{1}=-\frac{12\left(c^{2}-\omega^{2}\right)}{c^{2}} \frac{(2 d \Psi+B)\left(d^{2} \Psi+B d-E\right)}{\left(4 E \Psi+B^{2}\right)}, \quad B_{2}=\frac{12\left(c^{2}-\omega^{2}\right)}{c^{2}}$ $\frac{\left(d^{2} \Psi+B d-E\right)^{2}}{\left(4 E \Psi+B^{2}\right)}$,

where $\Psi=A-C, A, B, C$ and $E$ are free constants.

Using the values of the cluster one into equation (2.5) and equation (2.4), we obtain a new hyperbolic function solutions, rational function solutions and trigonometric function solutions, respectively:

$$
\begin{aligned}
& V_{1}(x, t)=-\frac{3\left(c^{2}-\omega^{2}\right)}{c^{2}}+\frac{3}{4} \frac{c^{2}-\omega^{2}}{c^{2}} \frac{4 E \Psi+B^{2}}{\Psi^{2}}\left(\frac{\sqrt{\Omega}}{2 \Psi} \tanh \left(\frac{\sqrt{\Omega} \xi}{2 A}\right)^{-2},\right. \\
& V_{2}(x, t)=-\frac{3\left(c^{2}-\omega^{2}\right)}{c^{2}}+\frac{3}{4} \frac{c^{2}-\omega^{2}}{c^{2}} \frac{4 E \Psi+B^{2}}{\Psi^{2}}\left(\frac{\sqrt{\Omega}}{2 \Psi} \operatorname{coth}\left(\frac{\sqrt{\Omega} \xi}{2 A}\right)^{-2},\right. \\
& V_{3}(x, t)=-\frac{3\left(c^{2}-\omega^{2}\right)}{c^{2}}+\frac{3}{4} \frac{c^{2}-\omega^{2}}{c^{2}} \frac{4 E \Psi+B^{2}}{\Psi^{2}}\left(\frac{-B}{2 \Psi}+\frac{\sqrt{\triangle}}{\Psi} \operatorname{tanth}\left(\frac{\sqrt{\triangle} \xi}{A}\right)^{-2},\right. \\
& V_{4}(x, t)=-\frac{3\left(c^{2}-\omega^{2}\right)}{c^{2}}+\frac{3}{4} \frac{c^{2}-\omega^{2}}{c^{2}} \frac{4 E \Psi+B^{2}}{\Psi^{2}}\left(\frac{-B}{2 \Psi}+\frac{\sqrt{\triangle}}{\Psi} \operatorname{coth}\left(\frac{\sqrt{\triangle} \xi}{A}\right)^{-2},\right. \\
& V_{5}(x, t)=-\frac{3\left(c^{2}-\omega^{2}\right)}{c^{2}}+\frac{3}{4} \frac{c^{2}-\omega^{2}}{c^{2}} \frac{4 E \Psi+B^{2}}{\Psi^{2}}\left(\frac{C_{2}}{C_{1}+C_{2} \xi}\right)^{-2}, \\
& V_{6}(x, t)=-\frac{3\left(c^{2}-\omega^{2}\right)}{c^{2}}+\frac{3}{4} \frac{c^{2}-\omega^{2}}{c^{2}} \frac{4 E \Psi+B^{2}}{\Psi^{2}}\left(-\frac{\sqrt{-} \Omega}{2 \Psi} \tan \left(\frac{\sqrt{-} \Omega \xi}{2 A}\right)^{-2},\right. \\
& V_{7}(x, t)=-\frac{3\left(c^{2}-\omega^{2}\right)}{c^{2}}+\frac{3}{4} \frac{c^{2}-\omega^{2}}{c^{2}} \frac{4 E \Psi+B^{2}}{\Psi^{2}}\left(\frac{\sqrt{-\Omega}}{2 \Psi} \cot \left(\frac{\sqrt{-} \Omega \xi}{2 A}\right)^{-2},\right. \\
& V_{8}(x, t)=-\frac{3\left(c^{2}-\omega^{2}\right)}{c^{2}}+\frac{3}{4} \frac{c^{2}-\omega^{2}}{c^{2}} \frac{4 E \Psi+B^{2}}{\Psi^{2}}\left(\frac{-B}{2 \Psi}-\frac{\sqrt{-} \triangle}{\Psi} \operatorname{tant}\left(\frac{\sqrt{-\triangle})^{-2}}{A}\right)^{-2}\right. \\
& V_{9}(x, t)=-\frac{3\left(c^{2}-\omega^{2}\right)}{c^{2}}+\frac{3}{4} \frac{c^{2}-\omega^{2}}{c^{2}} \frac{4 E \Psi+B^{2}}{\Psi^{2}}\left(\frac{-B}{2 \Psi}+\frac{\sqrt{-\triangle}}{\Psi} \cot \left(\frac{\sqrt{-\triangle} \xi}{A}\right)^{-2},\right.
\end{aligned}
$$


where $\xi=\frac{\beta A}{\omega}(x-\omega t)$.

Similarly, we could provide exact solutions, including the soliton solutions of other six case of the longitudinal wave motion equation in a magneto-electro-elastic circular rod, which are omitted for convenience. Here, we compare our obtained results with Xue's exact wave solution in [53]. Xue et al. only got one wave solution in [53]. In this paper, we received total sixty three exact wave solutions of equation (2.1).

Remark 1. All the above solutions of the longitudinal wave motion equation in a magneto-electro-elastic circular rod are merely novel and identical from the published in [53].

Remark 2. All of the above solutions of this article have been verified with symbolic computer program Maple through substituting them into the main equation as the longitudinal wave motion equation in a magneto-electro-elastic circular rod.

\subsection{Graphical illustrations of the obtained solutions}

For some suitable parameters, we get explicit travelling wave solution including compaction, bright solition, dark solition, and periodic wave solutions. These obtained solutions could assist in explaining the dynamics of spatiotemporal dispersion, higher-order dispersion, and full nonlinearity and also could be used to the propagation of solitons by a variety of waveguides. We illustrate some of the obtained solutions through choosing the proper values of the unknown free arbitrary parameters received. The graphical illustrations, including the three, two dimensional as well as the corresponding the contour structure surfaces of these obtained solutions are displayed in Figures 1, 2 and 3, respectively. Similarly, we could present the graphical description of the other six cases of the longitudinal wave motion equation in a magneto-electro-elastic circular rod, which are omitted for convenience.

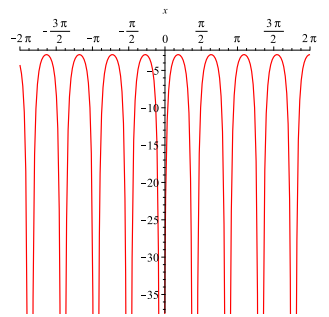

a.

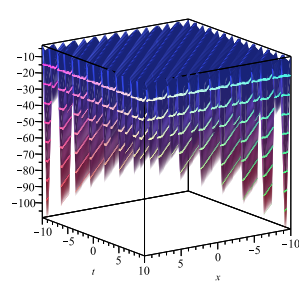

b.

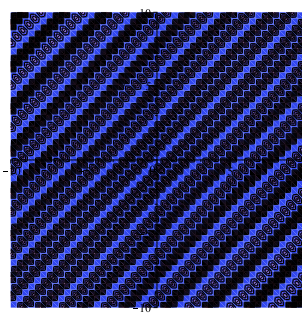

c.

FIGURE 1. $2 D, 3 D$ and contour plot graphical representation of solution in $V_{1}(x, t)$ and its projection at $t=2$ for the unknown perimeters $A=2, B=1, C=1, E=1, \omega=1, c=5.025$ and $p=1.25$. 


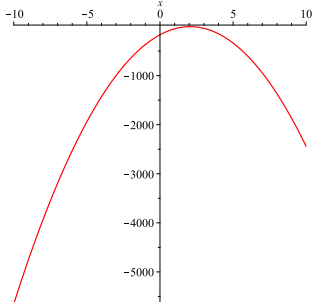

a.

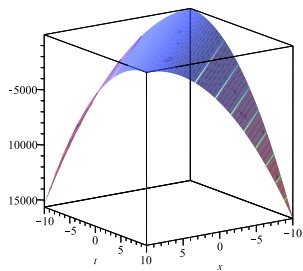

b.

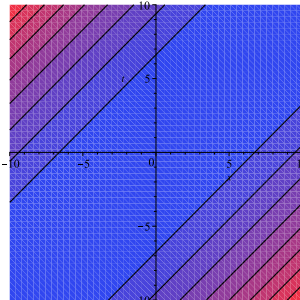

c.

FIGURE 2. $2 D, 3 D$ and contour plot graphical representation of solution in $V_{5}(x, t)$ and its projection at $t=2$ for the unknown perimeters $A=5, B=2, C=2, E=1, C_{1}=1, C_{2}=2, \omega=1, c=5.025$ and $p=1.25$.

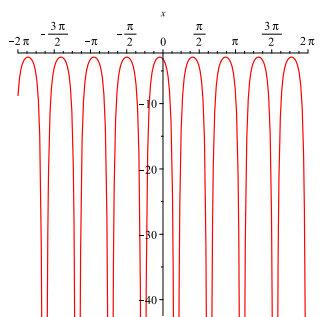

a.

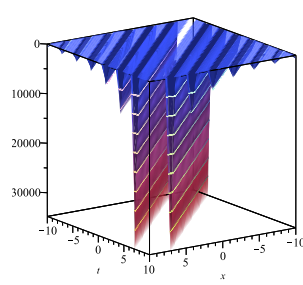

b.

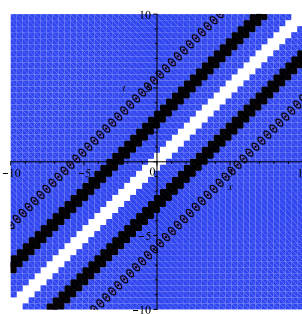

c.

FIGURE 3. $2 D, 3 D$ and contour plot graphical representation of solution in $V_{4}(x, t)$ and its projection at $t=2$ for the unknown perimeters $A=4, B=0, C=1, E=1, \omega=1, c=5.025$ and $p=1.25$.

\subsection{Results and Discussion}

In this section, we present the physical meaning of the new hyperbolic, trigonometric as well as rational function solutions achieved through the new generalized $\left(\frac{G \prime}{G}\right)$-expansion method on equation (2.1). These obtained explicit exact travelling wave solutions have proposed to the literature some relevant physical information about equation (2.1). It is considered that these explicit exact travelling solutions are linked to physical characteristics of hyperbolic functions $[18,50]$. In particular, the complex hyperbolic function solutions equation (2.13) and equation (2.14) attained in this paper are prognosticated to symbolize the gravitational potential of a cylinder modelled by equation (2.1) [50]. Conclusively, when it comes to the surfaces, Figures 1,2 and 3 , respectively have been plotted including the two, three-dimensional as well as the corresponding the contour plot surfaces via Maple 13 by studying 
proper values of parameters taken through the MEE material properties. When we examine all explicit exact travelling wave solutions achieved in this paper via the new generalized $\left(\frac{G \prime}{G}\right)$-expansion method, it is decided that two-dimensional, threedimensional surfaces and the corresponding contour plot surfaces are the graphical illustrations determined. Therefore, it can be announced that they are understandable physically because all the graphical illustrations dispense similar wave behaviours for proper values of parameters under the terms of magneto-electro-elastic material properties. Furthermore, it has been mentioned that all explicit exact travelling wave solutions satisfy equation (2.1) under the terms of equation (2.2) via Maple 13. To the skilled of our knowledge, the employment of the new generalized $\left(\frac{G \prime}{G}\right)$-expansion method to equation (2.1) has not been published beforehand. Similarly, we can give the explicit exact travelling wave solutions and all the graphical representation of the other six cases of equation (2.1), which are omitted for convenience.

\section{ExaCt SOlUtion OF THE DRINFELD-SOKOLOV-WILSON (DSW) EQUATION}

Drinfeld and Sokolov [25] and Wilson [51] has been proposed the DSW model for dispersive water waves and that play an important role in fluid dynamics [24,29]. To study of the model is given by

$$
\begin{array}{r}
\frac{\delta U}{\delta t}+\alpha_{1} V \frac{\delta V}{\delta x}=0, \\
\frac{\delta V}{\delta t}+\alpha_{2} \frac{\delta^{3} V}{\delta x^{3}}+\alpha_{3} U \frac{\delta V}{\delta x}+\alpha_{4} V \frac{\delta U}{\delta x}=0,
\end{array}
$$

where $\alpha_{1}, \alpha_{2}, \alpha_{3}$, and $\alpha_{4}$ are nonzero parameters. Recently, several researchers have been showed their interested on this model [1, 2, 13, 14, 21, 25, 27-32, 37, 40, 45, 51, 54, 59]. Qin and Yan [54] construct doubly periodic solutions of the coupled DSW equation by using an improve F-expansion method. Ayub et al. [14] have introduced the Exp-function method to look for solitary solutions of the generalized DSW system. Sweet and Gorder [45] applied the method of homotopy analysis to obtain analytical solutions of the generalized DSW system. Jawad [30] has introduced the traveling wave solutions and new solitary wave solutions of the DSW equation, Fornberg-Whitham equation, potential-TSF equation, Jimbo-Miwa equation, Modified Zakharov-Kuznetsov equation, and $(2+1)$-dimensional KonopelchenkoDubrovsky equation via the tanh and Sech function methods. Cesar [21] obtained exact solutions of this model by applying the improved tanh-coth. Abdelaziz and Ibrahim [1] has been proposed the enhanced of the $G^{\prime} / G$-expansion method combined with Liu's theorem to find new exact solutions of the nonlinear $(1+1)$ dimensional DSW equation. Zhang [59] established variational principles of the DSW equation Via the semi-inverse method and also obtained an exact solitary solution and exact singular periodic wave solution using the variational scheme. Gurefe 
and Misirli [28] applied the Exp-function method to obtain generalized solitary solutions of the generalized (2+1)dimensional Burgers-type equation and the generalized DSW system.

Niu and Liu [40] re-examined the well known coupled system as the DSW equation. Its proper Darboux transformation is constructed with the help of a Lax operator of fourth order and some solutions are calculated and a nonlinear superposition formula is worked out for the associated Backlund transformation. Arnous et al. [13] employed two integration schemes to draw solitons, singular periodic waves and other types of solutions of the DSW equation. Jin and $\mathrm{Lu}$ [31] applied the variational iteration method to solve the classical DSW equation. Hirota et al. [29] present this equation a novel type of solutions called static solitons and this static solutions interact with moving solitons without deformations.

In this section, we implemented the analytical method including the newly generalized $\left(\frac{G \prime}{G}\right)$-expansion method to solve exact solution of the Drinfeld-Sokolov-Wilson (DSW) equation.

Herein, we use traveling wave variable $V(\xi)=V(x, t), \xi=x+\beta t$. Then equation (3.1) and (3.2) converts into the following nonlinear ODEs:

$$
\begin{gathered}
\beta \frac{d U}{d \xi}+\alpha_{1} V \frac{d V}{d \xi}=0 \\
\beta \frac{d V}{d \xi}+\alpha_{2} \frac{d^{3} V}{d \xi^{3}}+\alpha_{3} U \frac{d V}{d \xi}+\alpha_{4} \frac{d U}{d \xi} V=0 .
\end{gathered}
$$

Now integrating equation (3.3), we have

$$
U=-\frac{\alpha_{1} V^{2}}{2 \beta}
$$

From equation (3.5) and equation (3.4), we obtain

$$
2 \beta \alpha_{2} \frac{d^{3} V}{d \xi^{3}}+2 \beta^{2} \frac{d V}{d \xi}-\alpha_{1}\left(\alpha_{3}+2 \alpha_{4}\right) V^{2} \frac{d V}{d \xi}=0 .
$$

Again integrating equation (3.6), we obtain

$$
2 \beta \alpha_{2} \frac{d^{2} V}{d \xi^{2}}+2 \beta^{2} V-\frac{\alpha_{1}\left(\alpha_{3}+2 \alpha_{4}\right)}{3} V^{3}=0 .
$$

According to the newly generalized $\left(\frac{G \prime}{G}\right)$-expansion method $[4,5,8]$, we obtain

$$
\mathrm{u}(\xi)=t_{0}+t_{1} S(\xi)+r_{1} S(\xi)^{-1},
$$

where $S(\xi)=(d+T(\xi))^{i}, T(\xi)=\left(\frac{G \prime}{G}\right)$ and the coefficients $t_{0}, t_{1}, r_{1}$ and $d$ are unknown costants. In view of equation (3.8) and equation (3.7), then equating each coefficients of $S(\xi)$ to zero, we have the following relations respectively:

- Phase one: $\beta=\frac{\left(B^{2}+4 E \Psi\right) \alpha_{2}}{4 A^{2}}, d=-\frac{B}{2 \Psi}, t_{0}=0, t_{1}=0, r_{1}=\frac{\alpha_{5} \alpha_{2}\left(B^{2}+4 E \Psi\right)}{\Psi A^{2}}$, where $\alpha_{5}= \pm \frac{3 B^{2}+12 E \Psi}{64 \alpha_{1} \alpha_{4}+32 \alpha_{1} \alpha_{3}}, \Psi=A-C, A, B, C$ and $E$ are free constants, 
- Phase two: $\beta=\frac{\left(B^{2}+4 E \Psi\right) \alpha_{2}}{A^{2}}, d=\alpha_{6}, t_{0}=-\frac{\alpha_{2} \alpha_{7}\left(B+\alpha_{6}\right)}{3 A^{2}}, t_{1}=0, r_{1}=$ $\frac{\left(B^{2}+4 E \Psi\right) \alpha_{2} \alpha_{7}}{\Psi A^{2}}$,

where $\alpha_{6}=\frac{-B \pm \sqrt{\left(B^{2}+36 E \Psi+8 B^{2}\right)}}{2}, \alpha_{7}= \pm \frac{24 B^{2}+96 E \Psi}{2 \alpha_{1} \alpha_{4}+\alpha_{1} \alpha_{3}}, \Psi=A-C, A, B, C$ and $E$ are free constants,

- Phase three: $\beta=\frac{\left(B^{2}+4 E \Psi\right) \alpha_{2}}{A^{2}}, d=d, t_{0}=-\frac{12 \alpha_{2}\left(8 E d \Psi^{2}+2 \Psi B^{2} d+4 B E \Psi+B^{3}\right)}{\left(\alpha_{3}+2 \alpha_{4}\right) \alpha_{7} A^{2} \alpha_{1}}$, $t_{1}=\frac{\left.\alpha_{7} \Psi \alpha_{2}\right)}{4 A^{2}}, r_{1}=0$, where $\Psi=A-C, A, B, C$ and $E$ are free constants.

For phase one, we get a new hyperbolic function solutions, rational function solutions and trigonometric function solutions to the DSW equation as given below, respectively:

$$
V_{11}(\xi)=\frac{\alpha_{5} \alpha_{2}\left(B^{2}+4 E \Psi\right)}{\Psi A^{2}} \frac{2 \Psi}{\sqrt{\Omega}} \tanh \left(\frac{\sqrt{\Omega} \xi}{2 A}\right),
$$

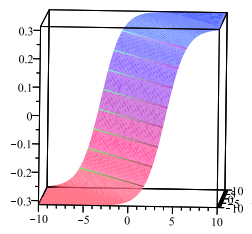

a



b.

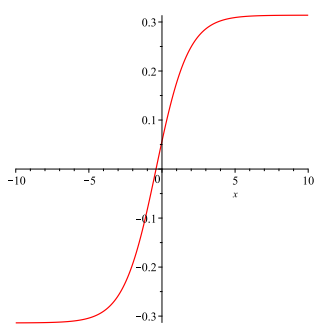

c.

FIgURE 4. $3 D$, contour plot and $2 D$ shape of the exact solution $V_{11}(x, t)$ at the particular values of $\alpha_{1}=1, \alpha_{2}=2, \alpha_{3}=3, \alpha_{4}=4$, $A=4, B=1, C=1, E=1$ and $t=1$.

$$
\begin{aligned}
& V_{12}(\xi)=\frac{\alpha_{5} \alpha_{2}\left(B^{2}+4 E \Psi\right)}{\Psi A^{2}} \frac{2 \Psi}{\sqrt{\Omega}} \operatorname{coth}\left(\frac{\sqrt{\Omega} \xi}{2 A}\right), \\
& V_{13}(\xi)=\frac{\alpha_{5} \alpha_{2}\left(B^{2}+4 E \Psi\right)}{\Psi A^{2}} \frac{2 \Psi}{\sqrt{\Omega}} \tan \left(\frac{\sqrt{-\Omega} \xi}{2 A}\right), \\
& V_{14}(\xi)=\frac{\alpha_{5} \alpha_{2}\left(B^{2}+4 E \Psi\right)}{\Psi A^{2}} \frac{2 \Psi}{\sqrt{\Omega}} \cot \left(\frac{\sqrt{-\Omega} \xi}{2 A}\right), \\
& V_{15}(\xi)=\frac{\alpha_{5} \alpha_{2}\left(B^{2}+4 E \Psi\right)}{\Psi A^{2}}\left(\frac{C_{2}}{C_{1}+C_{2} \xi}\right)^{-1}, \\
& V_{16}(\xi)=\frac{\alpha_{5} \alpha_{2}\left(B^{2}+4 E \Psi\right)}{\Psi A^{2}}\left(\frac{-B}{2 \Psi}+\frac{\sqrt{\triangle}}{\Psi} \operatorname{coth}\left(\frac{\sqrt{\triangle} \xi}{A}\right)\right)^{-1},
\end{aligned}
$$




$$
\begin{aligned}
& V_{17}(\xi)=\frac{\alpha_{5} \alpha_{2}\left(B^{2}+4 E \Psi\right)}{\Psi A^{2}}\left(\frac{-B}{2 \Psi}+\frac{\sqrt{\triangle}}{\Psi} \tanh \left(\frac{\sqrt{\triangle} \xi}{A}\right)\right)^{-1}, \\
& V_{18}(\xi)=\frac{\alpha_{5} \alpha_{2}\left(B^{2}+4 E \Psi\right)}{\Psi A^{2}}\left(\frac{-B}{2 \Psi}+\frac{\sqrt{-\triangle}}{\Psi} \cot \left(\frac{\sqrt{-\triangle} \xi}{A}\right)\right)^{-1}, \\
& V_{19}(\xi)=\frac{\alpha_{5} \alpha_{2}\left(B^{2}+4 E \Psi\right)}{\Psi A^{2}}\left(\frac{-B}{2 \Psi}+\frac{\sqrt{-\triangle}}{\Psi} \tan \left(\frac{\sqrt{-\triangle} \xi}{A}\right)\right)^{-1},
\end{aligned}
$$

where $\xi=x+\frac{\left(B^{2}+4 E \Psi\right) \alpha_{2}}{4 A^{2}} t$.

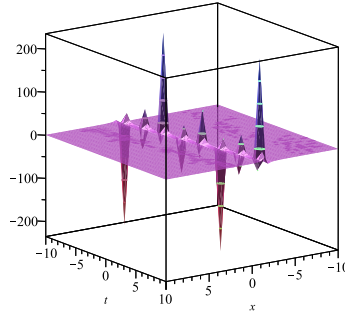

a.

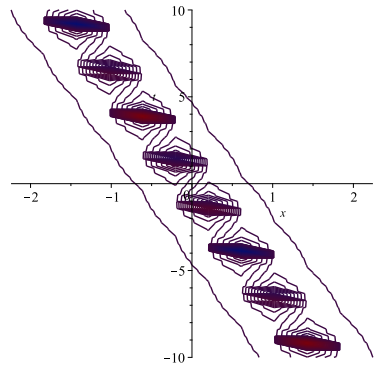

b.

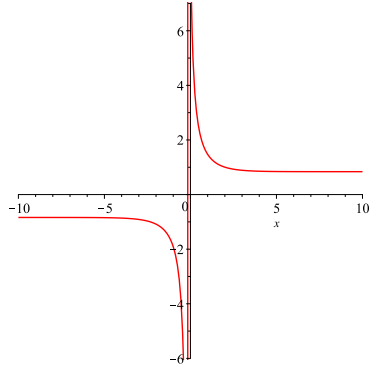

c.

FIgURE 5. $3 D$, contour plot and $2 D$ shape of the exact solution $V_{12}(x, t)$ at the particular values of $\alpha_{1}=1, \alpha_{2}=0.5, \alpha_{3}=0.3$, $\alpha_{4}=0.1, A=2, B=1, C=1, E=1$ and $t=1$.

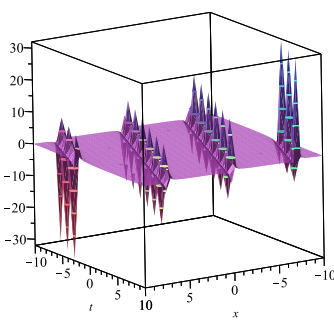

a.

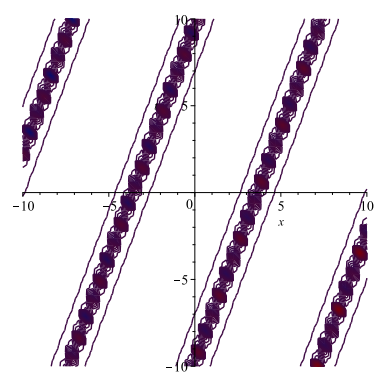

b.

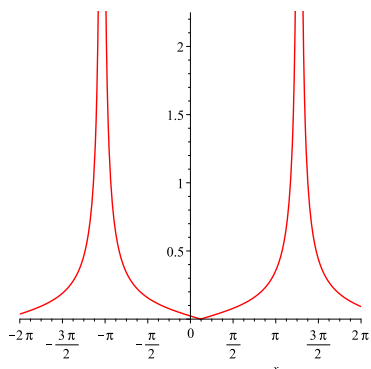

c.

FIgURE 6. $3 D$, contour plot and $2 D$ shape of the exact solution $V_{13}(x, t)$ at the particular values of $\alpha_{1}=1, \alpha_{2}=2, \alpha_{3}=3, \alpha_{4}=4$, $A=2, B=1, C=3, E=1$ and $t=1$.

Similarly to phase two, we have the following relations respectively:

$V_{21}(\xi)=-\frac{\alpha_{2} \alpha_{7}\left(B+\alpha_{6}\right)}{3 A^{2}}+\frac{\alpha_{5} \alpha_{2}\left(B^{2}+4 E \Psi\right)}{\Psi A^{2}}\left(\alpha_{6}+\frac{B}{2 \Psi}+\frac{\sqrt{\Omega}}{2 \Psi} \operatorname{coth}\left(\frac{\sqrt{\Omega} \xi}{2 A}\right)\right)^{-1}$, 


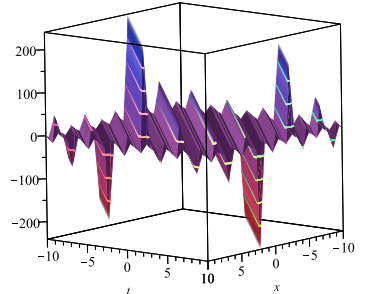

a.

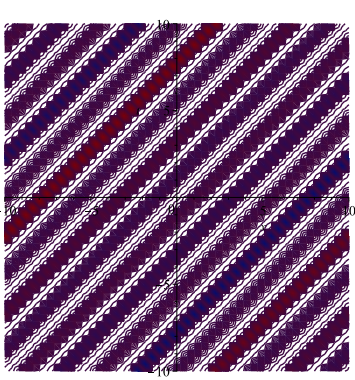

b.

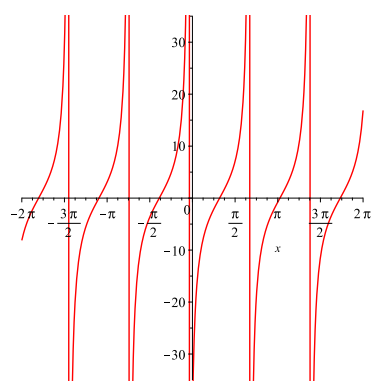

c.

FIGURE 7. $3 D$, contour plot and $2 D$ shape of the exact solution $V_{18}(x, t)$ at the particular values of $\alpha_{1}=1, \alpha_{2}=0.5, \alpha_{3}=0.3$, $\alpha_{4}=0.1, A=1, B=0, C=2, E=1$ and $t=1$.

$$
\begin{aligned}
& V_{22}(\xi)=-\frac{\alpha_{2} \alpha_{7}\left(B+\alpha_{6}\right)}{3 A^{2}}+\frac{\alpha_{5} \alpha_{2}\left(B^{2}+4 E \Psi\right)}{\Psi A^{2}}\left(\alpha_{6}+\frac{B}{2 \Psi}+\frac{\sqrt{\Omega})}{2 \Psi} \tanh \left(\frac{\sqrt{\Omega} \xi}{2 A}\right)\right)^{-1} \\
& V_{23}(\xi)=-\frac{\alpha_{2} \alpha_{7}\left(B+\alpha_{6}\right)}{3 A^{2}}+\frac{\alpha_{5} \alpha_{2}\left(B^{2}+4 E \Psi\right)}{\Psi A^{2}}\left(\alpha_{6}+\frac{B}{2 \Psi}+\frac{\sqrt{-\Omega}}{2 \Psi} \cot \left(\frac{\sqrt{-\Omega} \xi}{2 A}\right)\right)^{-1} \\
& V_{24}(\xi)=-\frac{\alpha_{2} \alpha_{7}\left(B+\alpha_{6}\right)}{3 A^{2}}+\frac{\alpha_{5} \alpha_{2}\left(B^{2}+4 E \Psi\right)}{\Psi A^{2}}\left(\alpha_{6}+\frac{B}{2 \Psi}+\frac{\sqrt{-\Omega}}{2 \Psi} \tan \left(\frac{\sqrt{-\Omega} \xi}{2 A}\right)\right)^{-1} \\
& V_{25}(\xi)=-\frac{\alpha_{2} \alpha_{7}\left(B+\alpha_{6}\right)}{3 A^{2}}+\frac{\alpha_{5} \alpha_{2}\left(B^{2}+4 E \Psi\right)}{\Psi A^{2}}\left(\alpha_{6}+\frac{B}{2 \Psi}+\frac{C_{2}}{C_{1}+C_{2} \xi}\right)^{-1} \\
& V_{26}(\xi)=-\frac{\alpha_{2} \alpha_{7}\left(B+\alpha_{6}\right)}{3 A^{2}}+\frac{\alpha_{5} \alpha_{2}\left(B^{2}+4 E \Psi\right)}{\Psi A^{2}}\left(\alpha_{6}+\frac{\sqrt{\triangle}}{\Psi} \tanh \left(\frac{\sqrt{\triangle} \xi}{A}\right)\right)^{-1} \\
& V_{27}(\xi)=-\frac{\alpha_{2} \alpha_{7}\left(B+\alpha_{6}\right)}{3 A^{2}}+\frac{\alpha_{5} \alpha_{2}\left(B^{2}+4 E \Psi\right)}{\Psi A^{2}}\left(\alpha_{6}+\frac{\sqrt{\triangle}}{\Psi} \operatorname{coth}\left(\frac{\sqrt{\triangle} \xi}{A}\right)\right)^{-1} \\
& V_{28}(\xi)=-\frac{\alpha_{2} \alpha_{7}\left(B+\alpha_{6}\right)}{3 A^{2}}+\frac{\alpha_{5} \alpha_{2}\left(B^{2}+4 E \Psi\right)}{\Psi A^{2}}\left(\alpha_{6}+\frac{\sqrt{-\triangle}}{\Psi} \tan \left(\frac{\sqrt{-\triangle} \xi}{A}\right)\right)^{-1} \\
& V_{29}(\xi)=-\frac{\alpha_{2} \alpha_{7}\left(B+\alpha_{6}\right)}{3 A^{2}}+\frac{\alpha_{5} \alpha_{2}\left(B^{2}+4 E \Psi\right)}{\Psi A^{2}}\left(\alpha_{6}+\frac{\sqrt{-\triangle}}{\Psi} \cot \left(\frac{\sqrt{-\triangle} \xi}{A}\right)\right)^{-1}
\end{aligned}
$$

where $\xi=x+\frac{\left(B^{2}+4 E \Psi\right) \alpha_{2}}{A^{2}} t$.

Again similarly to phase three, we find the following relations respectively:

$$
\begin{aligned}
& V_{31}(\xi)=-\frac{12 \alpha_{2}\left(8 E d \Psi^{2}+2 \Psi B^{2} d+4 B E \Psi+B^{3}\right)}{\left(\alpha_{3}+2 \alpha_{4}\right) \alpha_{7} A^{2} \alpha_{1}}+\frac{\alpha_{7} \Psi \alpha_{2}}{4 A^{2}}\left(d+\frac{B}{2 \Psi}+\frac{\sqrt{\Omega}}{2 \Psi} \tanh \left(\frac{\sqrt{\Omega} \xi}{2 A}\right)\right), \\
& \text { (3.27) } \\
& V_{32}(\xi)=-\frac{12 \alpha_{2}\left(8 E d \Psi^{2}+2 \Psi B^{2} d+4 B E \Psi+B^{3}\right)}{\left(\alpha_{3}+2 \alpha_{4}\right) \alpha_{7} A^{2} \alpha_{1}}+\frac{\alpha_{7} \Psi \alpha_{2}}{4 A^{2}}\left(d+\frac{B}{2 \Psi}+\frac{\sqrt{\Omega}}{2 \Psi} \operatorname{coth}\left(\frac{\sqrt{\Omega} \xi}{2 A}\right)\right),
\end{aligned}
$$






a.

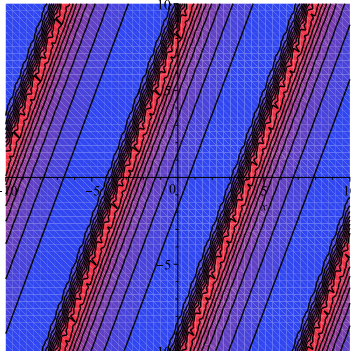

b.

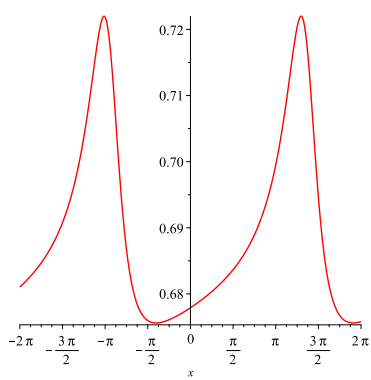

c.

FiguRE 8. $3 D$, contour plot and $2 D$ shape of the exact solution $V_{23}(x, t)$ at the particular values of $\alpha_{1}=1, \alpha_{2}=2, \alpha_{3}=3, \alpha_{4}=4$, $A=2, B=1, C=3, E=1$ and $t=1$.

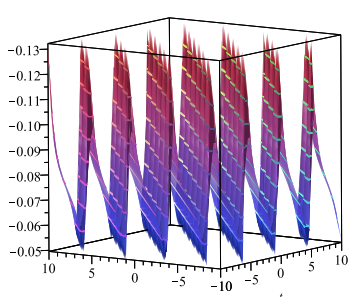

a.

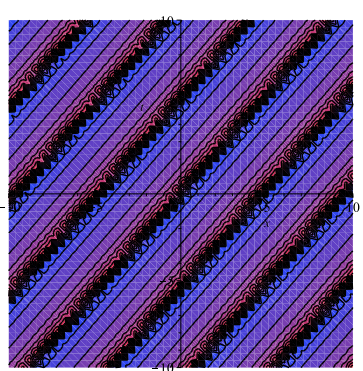

b.

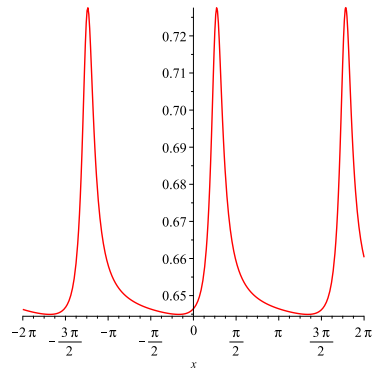

c.

FIgURE 9. $3 D$, contour plot and $2 D$ shape of the exact solution $V_{24}(x, t)$ at the particular values of $\alpha_{1}=1, \alpha_{2}=2, \alpha_{3}=3, \alpha_{4}=4$, $A=2, B=1, C=4, E=1$ and $t=1$.

$$
\begin{aligned}
& V_{33}(\xi)=-\frac{12 \alpha_{2}\left(8 E d \Psi^{2}+2 \Psi B^{2} d+4 B E \Psi+B^{3}\right)}{\left(\alpha_{3}+2 \alpha_{4}\right) \alpha_{7} A^{2} \alpha_{1}}+\frac{\alpha_{7} \Psi \alpha_{2}}{4 A^{2}}\left(d+\frac{B}{2 \Psi}+\frac{\sqrt{\Omega}}{2 \Psi} \tan \left(\frac{\sqrt{\Omega} \xi}{2 A}\right)\right), \\
& V_{34}(\xi)=-\frac{12 \alpha_{2}\left(8 E d \Psi^{2}+2 \Psi B^{2} d+4 B E \Psi+B^{3}\right)}{\left(\alpha_{3}+2 \alpha_{4}\right) \alpha_{7} A^{2} \alpha_{1}}+\frac{\alpha_{7} \Psi \alpha_{2}}{4 A^{2}}\left(d+\frac{B}{2 \Psi}+\frac{\sqrt{\Omega}}{2 \Psi} \cot \left(\frac{\sqrt{\Omega} \xi}{2 A}\right)\right), \\
& V_{35}(\xi)=-\frac{12 \alpha_{2}\left(8 E d \Psi^{2}+2 \Psi B^{2} d+4 B E \Psi+B^{3}\right)}{\left(\alpha_{3}+2 \alpha_{4}\right) \alpha_{7} A^{2} \alpha_{1}}+\frac{\alpha_{7} \Psi \alpha_{2}}{4 A^{2}}\left(d+\frac{B}{2 \Psi}+\frac{C_{2}}{C_{1}+C_{2} \xi}\right), \\
& V_{36}(\xi)=-\frac{12 \alpha_{2}\left(8 E d \Psi^{2}+2 \Psi B^{2} d+4 B E \Psi+B^{3}\right)}{\left(\alpha_{3}+2 \alpha_{4}\right) \alpha_{7} A^{2} \alpha_{1}}+\frac{\alpha_{7} \Psi \alpha_{2}}{4 A^{2}}\left(d+\frac{\sqrt{\triangle}}{\Psi} \operatorname{coth}\left(\frac{\sqrt{\triangle} \xi}{A}\right)\right),
\end{aligned}
$$




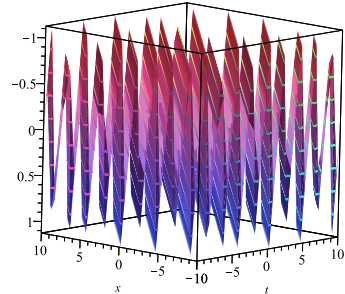

a.

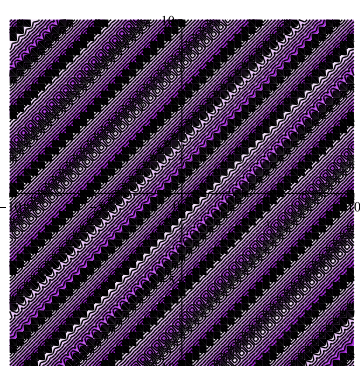

b.

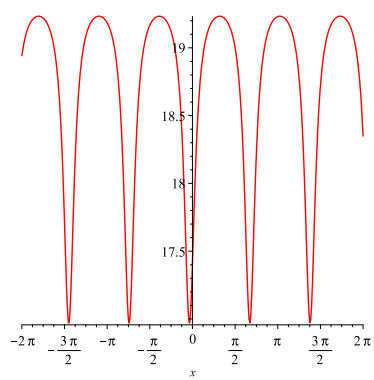

C.

FiguRE 10. $3 D$, contour plot and $2 D$ shape of the exact solution $V_{28}(x, t)$ at the particular values of $\alpha_{1}=1, \alpha_{2}=0.5, \alpha_{3}=0.3, \alpha_{4}=$ $0.1, A=1, B=0, C=2, E=2$ and $t=1$.

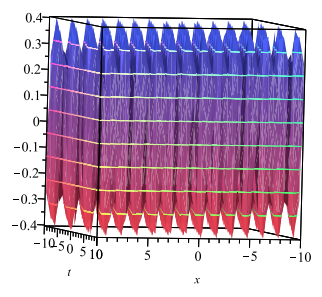

a.

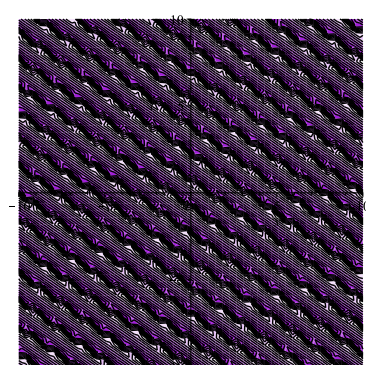

b.

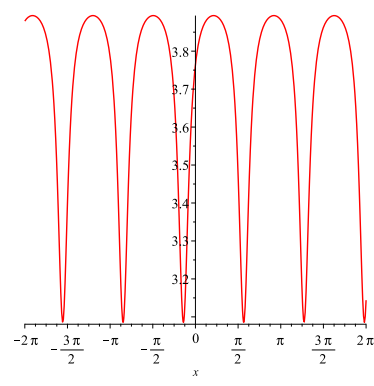

c.

FIgURE 11. $3 D$, contour plot and $2 D$ shape of the exact solution $V_{29}(x, t)$ at the particular values of $\alpha_{1}=1, \alpha_{2}=2, \alpha_{3}=3, \alpha_{4}=4$, $A=1, B=0, C=2, E=2$ and $t=1$.

$$
V_{37}(\xi)=-\frac{12 \alpha_{2}\left(8 E d \Psi^{2}+2 \Psi B^{2} d+4 B E \Psi+B^{3}\right)}{\left(\alpha_{3}+2 \alpha_{4}\right) \alpha_{7} A^{2} \alpha_{1}}+\frac{\alpha_{7} \Psi \alpha_{2}}{4 A^{2}}\left(d+\frac{\sqrt{\triangle}}{\Psi} \tanh \left(\frac{\sqrt{\triangle} \xi}{A}\right)\right),
$$$$
V_{38}(\xi)=-\frac{12 \alpha_{2}\left(8 E d \Psi^{2}+2 \Psi B^{2} d+4 B E \Psi+B^{3}\right)}{\left(\alpha_{3}+2 \alpha_{4}\right) \alpha_{7} A^{2} \alpha_{1}}+\frac{\alpha_{7} \Psi \alpha_{2}}{4 A^{2}}\left(d+\frac{\sqrt{-\triangle}}{\Psi} \cot \left(\frac{\sqrt{-\triangle} \xi}{A}\right)\right),
$$

$$
V_{39}(\xi)=-\frac{12 \alpha_{2}\left(8 E d \Psi^{2}+2 \Psi B^{2} d+4 B E \Psi+B^{3}\right)}{\left(\alpha_{3}+2 \alpha_{4}\right) \alpha_{7} A^{2} \alpha_{1}}+\frac{\alpha_{7} \Psi \alpha_{2}}{4 A^{2}}\left(d+\frac{\sqrt{-\triangle}}{\Psi} \tan \left(\frac{\sqrt{-\triangle} \xi}{A}\right)\right),
$$

where $\xi=x+\frac{\left(B^{2}+4 E \Psi\right) \alpha_{2}}{A^{2}} t$. 
Remark 3. All of the above solutions of this article have been verified with symbolic computer program Maple through substituting them into the main equation as the DSW equation.

\subsection{Graphical illustrations of the obtained solutions}

Similarly, some figures of the solutions are presented in the Figures 4, 5, 6, 7, 8, 9, 10 and 11 , respectively.

\section{Conclusion}

In this research, the analytical soliton solutions to the considered model have been successfully got through the novel generalized $\left(\frac{G \prime}{G}\right)$-expansion method. The solutions are obtained by rational, trigonometric and hyperbolic function by using this method. The obtained solutions showed that the studied method is more effective than the other methods (e.g., exp-function method, the Jacobi elliptic function expansion method), because it gives more new solutions. Consequently, the conclusions confirm that the above method is useful algorithms for the analytical approach of a broad spectrum of nonlinear modes of NLEEs arising in mathematical physics. We may decide that the proposed method could be continued to determine the nonlinear problems that appear in the theory of solitons and other fields.

\section{REFERENCES}

[1] M. A. M. Abdelaziz and M. E. A. Ibrahim, "Enhanced $\left(G^{\prime} / G\right)$-Expansion Method and Its Application to the Drinfeld-Sokolov-Wilson Equation," Phys. Sci. Inter. J., vol. 9, no. 1, pp. 1-22, 2016.

[2] M. Abdelrahman, M. Sohaly, and O. Moaaz, "The deterministic and stochastic solutions of the NLEEs in mathematical physics," Int. J. Appl. Comput. Math., vol. 5, no. 2, p. 40, 2019, doi: 10.1007/s40819-019-0623-1.

[3] M. N. Alam, "Exact solutions to the foam drainage equation by using the new generalized $\left(G^{\prime} / G\right)$ expansion method," Results in Phys., vol. 5, pp. 168-177, 2015, doi: 10.1016/j.rinp.2015.07.001.

[4] M. N. Alam and M. A. Akbar, "A new $\left(G^{\prime} / G\right)$-expansion method and its application to the Burgers equation," Walailak J. Sci. Tech., vol. 11, no. 8, pp. 643-658, 2014, doi: 10.14456/WJST.2014.86.

[5] M. N. Alam and M. A. Akbar, "The new approach of generalized $\left(G^{\prime} / G\right)$-Expansion Method for nonlinear evolution equations," Ain Shams Eng. J., vol. 5, no. 2, pp. 595-603, 2014, doi: 10.1016/j.asej.2013.12.008.

[6] M. N. Alam, M. A. Akbar, and M. Hoque, "Exact traveling wave solutions of the $(3+1)$ dimensional mKdV-ZK equation and the $(1+1)$-dimensional compound KdVB equation using new approach of the generalized $\left(G^{\prime} / G\right)$-expansion method,' Pramana J. Phys., vol. 83, no. 3, pp. 317-329, 2014, doi: 10.1007/s12043-014-0776-8.

[7] M. N. Alam, M. A. Akbar, and S. Mohyud-Din, "A novel $\left(G^{\prime} / G\right)$-expansion method and its application to the Boussinesq equation," Chin. Phys. B, vol. 23, no. 2, p. 020203, 2014, doi: 10.1088/1674-1056/23/2/020203.

[8] M. N. Alam, M. Akbar, and S. Mohyud-Din, "General traveling wave solutions of the strain wave equation in microstructured solids via the new approach of generalized $\left(G^{\prime} / G\right)$-Expansion method," Alexandria Eng. J., vol. 53, pp. 233-241, 2014, doi: http://dx.doi.org/10.1016/j.aej.2014.01.002. 
[9] M. N. Alam and M. M. Alam, "An analytical method for solving exact solutions of a nonlinear evolution equation describing the dynamics of ionic currents along microtubules," J. Taibah Univer. Sci., vol. 11, no. 6, pp. 939-948, 2017, doi: 10.1016/j.jtusci.2016.11.004.

[10] M. N. Alam and F. B. M. Belgacem, "Microtubules nonlinear models dynamics investigations through the $\exp (-\phi(\xi))$-expansion method implementation," Mathematics, vol. 4, no. 1, p. 6, 2016, doi: 10.3390/math4010006.

[11] M. N. Alam and X. Li, "Exact traveling wave solutions to higher order nonlinear equations," $J$. Ocean Eng. Sci., vol. 4, no. 3, pp. 276-288, 2019, doi: 10.1016/j.joes.2019.05.003.

[12] M. N. Alam and C. Tunc, "An analytical method for solving exact solutions of the nonlinear Bogoyavlenskii equation and the nonlinear diffusive predator-prey system," Alexandria Eng. J., vol. 55, no. 2, pp. 1855-1865, 2016, doi: 10.1016/j.aej.2016.04.024.

[13] A. H. Arnous, M. Mirzazadeh, and M. Eslami, "Exact solutions of the Drinfeld-Sokolov-Wilson equation using Bäcklund transformation of Riccati equation and trial function approach," Pramana J. Phys., vol. 86, no. 6, pp. 1153-1160, 2016, doi: 10.1007/s12043-015-1179-1.

[14] K. Ayub, Q. Ul-Hassan, and S. T. Mohyud-Din, "Some new solutions of generalized DrinfeldSokolov-Wilson system using Exp-function method," Inter. J. Phys. Sci., vol. 2, no. 6, pp. 089094, 2014.

[15] H. M. Baskonus and H. Bulut, "Exponential prototype structure for $(2+1)$-dimensional BoitiLeon-Pempinelli systems in mathematical physics," Waves Random Complex Media, vol. 26, no. 2, pp. 189-196, 2016, doi: 10.1080/17455030.2015.1132860.

[16] H. M. Baskonus, H. Bulut, and A. Atangana, "On the complex and hyperbolic structures of longitudinal wave equation in a magneto-electro-elastic circular rod," Smart Mater. Struct., vol. 25, no. 3, p. 035022, 2016, doi: 10.1088/0964-1726/25/3/035022.

[17] H. M. Baskonus, T. A. Sulaiman, and H. Bulut, "On the novel wave behaviors to the coupled nonlinear Maccari's system with complex structure," Optik, vol. 131, pp. 1036-1043, 2017, doi: 10.1016/j.ijleo.2016.10.135.

[18] W. H. Beyer, CRC Standard Mathematical Tables 28th ed. New York: Boca Raton, 1987.

[19] H. Bulut, T. A. Sulaiman, and H. M. Baskonus, "New solitary and optical wave structures to the Korteweg de Vries equation with dual-power law nonlinearity," Opt. Quant. Electron., vol. 48, p. 564, 2016, doi: 10.1007/s11082-016-0831-4.

[20] H. Bulut, T. A. Sulaiman, and H. M. Baskonus, "On the solitary wave solutions to the longitudinal wave equation in MEE circular rod," Opt. Quant. Electron, vol. 50, p. 87, 2018, doi: 10.1007/s11082-018-1362-y.

[21] A. G. Cesar, "Generalized Drinfeld-Sokolov-Wilson Equation: Exact Solutions," Adv. Studies in Theoretical Phys., vol. 4, no. 12, pp. 585-591, 2017, doi: 10.12988/astp.2017.7943.

[22] J. Y. Chen, E. Pan, and H. L. Chen, "Wave propagation in magnetoelectro-elastic multilayered plates," Int. J. Solids Struct., vol. 44, no. 3-4, pp. 1073-1085, 2007, doi: 10.1016/j.ijsolstr.2006.06.003.

[23] P. Chen and Y. Shen, "Propagation of axial shear magnetoelectro-elastic waves in piezoelectricpiezomagnetic composites with randomly distributed cylindrical inhomogeneities," Int. J. Solids Struct., vol. 44, no. 3-4, pp. 1511-1532, 2007, doi: 10.1016/j.ijsolstr.2006.06.037.

[24] V. G. Drinfeld and V. V. Sokolov, "Lie algebras and equations of Korteweg-de Vries type," J. Sov. Math., vol. 30, p. 1975, 1985, doi: 10.1007/BF02105860.

[25] V. Drinfeld and V. Sokolov, "Equations of Korteweg-de Vries type, and simple Lie algebras," (Russian) Dokl. Akad. Nauk SSSR, vol. 258, no. 1, pp. 11-16, 1981.

[26] J. K. Du, Y. P. Shen, D. Y. Ye, and F. R. Yue, "Scattering of anti-plane shear waves by a partially debonded magneto-electo-elastic circular cylindrical inhomogeneity," Int. J. Eng. Sci., vol. 42, no. 8-9, pp. 887-913, 2004, doi: 10.1016/j.ijengsci.2003.07.010. 
[27] E. Fan, "An algebraic method for finding a series of exact solutions to integrable and nonintegrable nonlinear evolution equations," J. Phys. A, vol. 36, no. 25, pp. 7009-7026, 2003, doi: 10.1088/0305-4470/36/25/308.

[28] Y. Gurefe and E. Misirli, "Exp-function method for solving nonlinear evolution equations with higher order non linearity," Appl. Math. Sci. (Ruse), vol. 61, no. 8, pp. 2025-2030, 2011, doi: 10.1016/j.camwa.2010.08.060.

[29] R. Hirota, B. Grammaticos, and A. Ramani, "Soliton structure of the Drinfeld-Sokolov-Wilson equation," J. Math. Phys., vol. 27, no. 6, pp. 1499-1505, 1986, doi: 10.1063/1.527110.

[30] A. J. M. Jawad, "New Solitary Wave Solutions for Some Non-linear Partial Differential Equations," Inter. J. Innovat. Resear. Sci. Eng., vol. 4, no. 12, p. 162, 2016.

[31] L. Jin and J. Lu, "Variational Iteration Method for the Classical Drinfeld-Sokolov-Wilson," Thermal Sci., vol. 18, no. 5, pp. 1543-1546, 2014, doi: 10.2298/TSCI1405543J.

[32] U. Khan, R. Ellahi, R. Khan, and S. T. Mohyud-Din, "Extracting new solitary wave solutions of Benny-Luke equation and Phi-4 equation of Fractional Order by using $\left(G^{\prime} / G\right)$-expansion Method," Opt. Quant. Electron., vol. 49, p. 362, 2017, doi: 10.1007/s11082-017-1191-4.

[33] X. Ma, Y. Pan, and L. Chang, "Explicit travelling wave solutions in a magneto-electro-elastic circular rod," Inter. J. Comput. Sci., vol. 10, no. 1, p. 3, 2013.

[34] N. P. Maity and R. Maity, "Surface plasmon waves on noble metals at optical wavelengths," Int. J. Comput. Sci., vol. 8, no. 3, pp. 485-490, 2011.

[35] W. Malfliet, "Solitary wave solutions of nonlinear wave equation," Amer. J. Phys., vol. 60, no. 7, pp. 650-654, 1992, doi: 10.1119/1.17120.

[36] A. Milazzo and C. Orlando, "A beam finite element for magneto-electo-elastic multilayered composite structures," Int. J. Eng. Sci., vol. 94, no. 12, pp. 3710-3721, 2012, doi: 10.1016/j.compstruct.2012.06.011.

[37] E. Misirli and Y. Gurefe, "Exact solutions of the Drinfeld-Sokolov-Wilson equation using the exp-function method," Appl. Math. Comput., vol. 216, no. 9, pp. 2623-2627, 2010, doi: 10.1016/j.amc.2010.03.105.

[38] S. T. Mohyud-Din and A. Irshad, "Solitary wave solutions of some nonlinear PDEs arising in electronics,” Opt. Quant. Electron., vol. 49, p. 130, 2017, doi: 10.1007/s11082-017-0974-y.

[39] H. Naher and F. A. Abdullah, "New approach of $\left(G^{\prime} / G\right)$-expansion method and new approach of generalized $\left(G^{\prime} / G\right)$-expansion method for nonlinear evolution equation," AIP Advances, vol. 3, p. 032116, 2013, doi: 10.1063/1.4794947.

[40] X. X. Niu and Q. P. Liu, "Darboux Transformation for Drinfeld-Sokolov-Wilson Equation," Commun. Theor. Phys. (Beijing), vol. 64, no. 5, pp. 491-494, 2015, doi: 10.1088/0253-6102/64/5/491.

[41] F. Ozpinar, H. M. Baskonus, and H. Bulut, "On the complex and hyperbolic structures for the $(2+1)$-dimensional Boussinesq water equation,” Entropy, vol. 17, no. 12, pp. 8267-8277, 2015, doi: 10.3390/e17127878.

[42] N. Pakam and A. Arockiarajan, "An analytical model for predicting the effective properties of magneto-electo-elastic composities," Comput. Matter. Sci., vol. 65, pp. 19-28, 2012, doi: 10.1016/j.commatsci.2012.07.003.

[43] Y. J. Ren and H. Q. Zhang, "A generalized F-expansion method to find abundant families of Jacobi elliptic function solutions of the $(2+1)$-dimensional Nizhnik-Novikov-Veselov equation," Chaos Solitons Fractals, vol. 27, no. 4, pp. 959-979, 2006, doi: 10.1016/j.chaos.2005.04.063.

[44] A. R. Seadawy and J. Manafian, "New soliton solution to the longitudinal wave equation in a Magneto-electro-elastic circular rod," Results in Phys., vol. 8, pp. 1158-1167, 2018, doi: 10.1016/j.rinp.2018.01.062.

[45] E. Sweet and R. Gorder, "Analytical solutions to a generalized Drinfeld-Sokolov equation related to DSSH and KdV6," Appl. Math. Comput., vol. 216, no. 10, pp. 2783-2791, 2010, doi: 10.1016/j.amc.2010.03.128. 
[46] M. L. Wang, J. L. Zhang, and X. Z. Li, "The $\left(G^{\prime} / G\right)$-expansion method and travelling wave solutions of nonlinear evolutions equations in mathematical physics," Phys. Lett. A, vol. 372, no. 4, pp. 417-423, 2008, doi: 10.1016/j.physleta.2007.07.051.

[47] A. M. Wazwaz, "A sine-cosine method for handling nonlinear wave equations," Math. Comput. Modelling, vol. 40, no. 5-6, pp. 499-508, 2004, doi: 10.1016/j.mcm.2003.12.010.

[48] A. M. Wazwaz, "The tanh method for travelling wave solutions of nonlinear equations," Appl. Math. Comput., vol. 154, no. 3, pp. 714-723, 2004, doi: 10.1016/S0096-3003(03)00745-8.

[49] A. M. Wazwaz, "New (3+1)-dimensional nonlinear evolution equations with mKdV equation constituting its main part: Multiple soliton solutions," Chaos Solitons Fractals, vol. 76, no. 4, pp. 93-97, 2015, doi: 10.1016/j.chaos.2015.03.018.

[50] E. W. Weisstein, CRC Concise Encyclopedia of Mathematics. Second Edition. New York: Boca Raton, FL, 2003.

[51] G. Wilson, "The affine lie algebra $C_{2}^{1}$ and an equation of Hirota and Satsuma," Phys. Lett. A, vol. 89, no. 7, pp. 332-334, 1982, doi: 10.1016/0375-9601(82)90186-4.

[52] B. Wu, J. G. Yu, and C. F. He, "Wave propagation in nonhomogeneous magneto-electro-elastic plates," J. Sound Vib., vol. 317, no. 1, pp. 250-264, 2008, doi: 10.1016/j.jsv.2008.03.008.

[53] C. X. Xue, E. Pan, and S. Y. Zhang, "Solitary waves in a magnetoelectro-elastic circular rod," Smart Mater. Struct., vol. 20, pp. 105 010-17, 2011, doi: 10.1088/0964-1726/20/10/105010.

[54] Z. Xue-Qin and Z. Hong-Yan, "An Improved F-Expansion Method and Its Application to Coupled Drinfeld-Sokolov-Wilson Equation,” Commun. Theor. Phys. (Beijing), vol. 50, no. 2, pp. 309-314, 2008, doi: 10.1088/0253-6102/50/2/05.

[55] C. Yan, "A simple transformation for nonlinear waves," Phys. Lett. A, vol. 224, no. 1-2, pp. 77-84, 1996, doi: 10.1016/S0375-9601(96)00770-0.

[56] M. Younis and S. Ali, "Bright, dark and singular solitons in magneto-electro-elastic circular rod," Waves Random Complex Media, vol. 25, no. 4, pp. 549-555, 2015, doi: 10.1080/17455030.2015.1058993.

[57] J. Zhang, F. Jiang, and X. Zhao, "An improved $\left(G^{\prime} / G\right)$-expansion method for solving nonlinear evolution equations," Inter. J. Comput. Math., vol. 87, no. 8, pp. 1716-1725, 2010, doi: $10.1080 / 00207160802450166$.

[58] J. Zhang, X. Wei, and Y. Lu, “A generalized $\left(G^{\prime} / G\right)$-expansion method and its applications,” Opt. Quant. Electron., vol. 372, no. 20, pp. 3653-3658, 2008, doi: 10.1016/j.physleta.2008.02.027.

[59] J. L. Zhang, M. L. Wang, Y. M. Wang, and Z. D. Fang, "The improved F-expansion method and its applications," Phys. Lett. A, vol. 350, no. 1-2, pp. 103-109, 2006, doi: 10.1016/j.physleta.2005.10.099.

\section{Authors' addresses}

\section{Md Nur Alam}

School of Mathematical Sciences, University of Science and Technology of China, 230026 Hefei, China, and Department of Mathematics, Pabna University of Science and Technology, 6600 Pabna, Bangladesh

E-mail address: nuralam23@mail.ustc.edu.cn, nuralam.pstu23@gmail.com

\section{Cemil Tunc}

Department of Mathematics, Faculty of Sciences, Van Yuzuncu Yil University, 65080 Van, Turkey

E-mail address: cemtunc@yahoo.com 